\title{
New pancrelipase is safe and effective
}

A new delayed-release formulation of pancrelipase $\left(\mathrm{CREON}^{\circledR}\right.$, Abbott Products, Abbott Park, IL, USA) seems to be an effective treatment for patients with maldigestion owing to exocrine pancreatic insufficiency (EPI) as a result of chronic pancreatitis or pancreatic surgery.

"The new pancrelipase significantly improved the digestion and absorption of dietary fat [versus placebo]," comments David Whitcomb, corresponding author of this multinational study. Abdominal symptoms, such as stool frequency, diarrhea, abdominal pain and flatulence improved in the actively treated group and adverse events were minimal. "[Unexpectedly], the new pancrelipase formula also improved protein absorption," Whitcomb continues.

The 54 participants had severe EPI (coefficient of fat absorption $<80 \%$ and/or stool fat excretion $\geq 40 \mathrm{~g}$, while taking placebo and eating a high-fat diet). These patients were randomly assigned to
1 week of either placebo or pancrelipase (72,000 lipase units with each of three main meals and 36,000 lipase units with each of two snacks daily).

\section{4 ...the new pancrelipase formula also improved protein absorption... 77}

"The importance of pancrelipase in improving nutrition, and especially protein digestion, has not been adequately studied," notes Whitcomb. He suggests that undertreatment, rather than alcoholism, might account for some of the malnutrition seen in patients with EPI owing to chronic pancreatitis.

\section{Caroline Barranco}

Original article Whitcomb, D. C. et al. Pancrelipase delayed-release capsules (CREON) for exocrine pancreatic insufficiency due to chronic pancreatitis or pancreatic surgery: a double-blind randomized trial. Am. J. Gastroenterol. 105, 2276-2286 (2010) 